\title{
Coalition Formation for Cooperative Information Agent-Based Systems
}

\author{
Nacer eddine Zarour, Sabrina Bouzidi
}

\begin{abstract}
The communication technology evolution led to an increase of the carried out services' and tasks' number. The aim of actual research in the cooperation and particularly the negotiation between agents is to reach a coherent global state of the multiagent system by favoring agents' synergy. In this paper, we propose a coalition formation-based negotiation model for the task allocation in the cooperative information agent-based systems. In this model, the agent that activates a negotiation seeks partners for achieving a complex task. The way that the partners take part in a coalition is done one by one according to the choice of all the coalition members. This choice is based on a multicriterion analysis. Some obtained experimental results show the suggested model performances.
\end{abstract}

Keywords: Negotiation, coalition formation, cooperative information systems, multiagent systems, task allocation.

\section{Introduction}

In an open environment as Cooperative Information Systems (CISs), which gathers distributed; heterogeneous; and autonomous Information Systems (ISs), the cooperation requires the intervention of a negotiation process in order to identify and structure the various problems, to propose and defend the solutions, to re-examine the intentions in a conflict, and finally to confirm the commitments [1]. In our context, each IS is modelled by an autonomous, rational, and egoistic agent.

Several negotiation models were developed like Market mechanisms [2], Contract Net Protocol [3], Social Laws [4], MultiAgent Planification [5], and Organizational structures (coalitions, teams, congregations, etc.) [6]. The coalition formation is a negotiation technique which is particularly preferred for solving the conflicts between agents whose behavior is economic and thus rational. A coalition is a short-term organization based on specific and contextual commitments of involving agents. This allows agents to profit from their respective competencies. To solve the task allocation problem, several coalition formation-based models were suggested [7] [8] [3]. However, these models are not adapted to the context of CISs because they do not define relations of partnership between agents, but rather relations of subcontracting. Generally in these models, the agent that activates a negotiation process will only decides to structure the coalition. It selects its partners separately according to its own desires. Consequently, the agents' autonomy is not respected.

The objective of this work is to propose a coalition formation-based model for the task allocation in CISs. The proposed model, named CFoTACIS ${ }^{1}$, is inspired from the reality of the cooperation between entreprises. It respects the agents' autonomy by allowing them to take part in the coalition formation process. It is recursive and includes four phases, the initialization, the negotiation, the evaluation, and the finalization.

The rest of the paper is organized as follows. In the following section, we present some related work to the coalition formation. In section 3, we develop the suggested negotiation model. Section 4 enumerates the proposed model properties. Some experimental results are presented in section 5. Section 6 summarizes the contributions of this paper and the research perspectives.

\footnotetext{
${ }^{1}$ Coalition Formation for Task Allocation in Cooperative Information Systems.
} 


\section{Related work}

According to the literature, we consider two approaches for studying the coalition formation process, the macroscopic approach and the microscopic one. The macroscopic approach, which is based on the game theory, considers the coalition as the basic unit [9]. The microscopic approach considers agents as the basic unit where several models were proposed and applied in many fields, especially the e-business [10] and the task allocation [7] [8] [3].

We focuss our discussion on the models based on the second approach which deal with the task allocation problem. In [7], the authors proposed a model where agents exchange their preferences w.r.t. the possible solutions. Although, forcing agents to form alliances ensures the termination of the negotiation process, but the model does not respect agents' freedom since it imposes a solution which may not satisfy all agents.

In [8], the authors propose a model where the agent that activates the negotiation process first filters agents of its environment. It uses a multicriterion analysis to find agents that best satisfy its requirements. Then, it starts an argumentative negotiation with the candidates, which are classified according to its preferences. This approach does not ensure the reach of a consensus but limiting the negotiation time seems to be a reasonable strategy.

Two models are proposed in [3]. The first one is dedicated to the competitive agents. The agent selection is based on a preference model, which is built using several criteria. The major inconvenient of this model is that the representing agent of the coalition has a kind of authority on the coalition members. In the second model, agents cooperate in an altruistic way. The model ensures the reach of a consensus and has some advantages like the integration of the new partners one by one in the coalition. However, the altruistic strategy of agents does not require the gradual integration of agents in the coalition. This approach seems to be interesting in the case of egoistic agents. In [10], the authors proposed a formalization of the trust criteria which an agent grants to the other ones of the environment. This trust modeling ensures the stability in the coalition formation due to its reliability. However, the authors do not specify how to find the value of the current evaluation of trust. In order to response at this question in our proposal, we will deduce the trust from some criteria.

\section{CFoTACIS: A coalition formation-based negotiation model in CISs}

Before describing the suggested model, let us admit the following assumption: an agent which wants to integrate the CIS must update the portal of this CIS [4]. It must specify its localization, its know-how, the information about its branch of industry, its competencies, its capital, etc.

CFoTACIS is recursive and includes four phases, the initialization, the negotiation, the evaluation, and the finalization. In the beginning of the negotiation process, the manager which is the first coalition member, follows only the model phases. After that, when new partners join the coalition and become members, all the members try to find the next partner. The negotiation process iters until the coalition size will be reached. Let us give the essential description of the different phases

\subsection{The initialization phase}

The manager consults the portal of the CIS (first assumption) to obtain useful informations on agents. Then, it decomposes the global task into subtasks w.r.t. the agents' industry branches. For each subtask, the manager makes a set of the candidates among agents of the environment. 


\subsection{The negotiation phase}

The manager simultaneously sends the proposals to agents of the same set. The message which the manager sends to the candidates must contain all information about the cooperation project, as well as the waiting period that accords to them. Then, the manager receives the responses from the candidates. Figure 1 represents the state transitions for a negotiation between a manager $(m)$ and a candidate $(c)$. After receiving a proposal from a manager for cooperation, a candidate can:

- refuse to cooperate (refuse $(c, m))$;

- accept to achieve the subtask (accept $(c, m))$;

- accept to achieve the subtask and also propose to achieve another subtask $(s)(o k(c, m))$;

- refuse to achieve the subtask but propose to achieve another subtask(s) (no(c,m));

- not answer. In this case, after the expiry of a waiting period, the manager considers the not reply as a rejection (refuse $(c, m)$ ).

If the candidate agrees to cooperate, it must send to the manager a message that contains its consideration of the cost and the time for achieving the subtask, as well as the maximum reply time that it grants to the manager to evaluate the proposal.

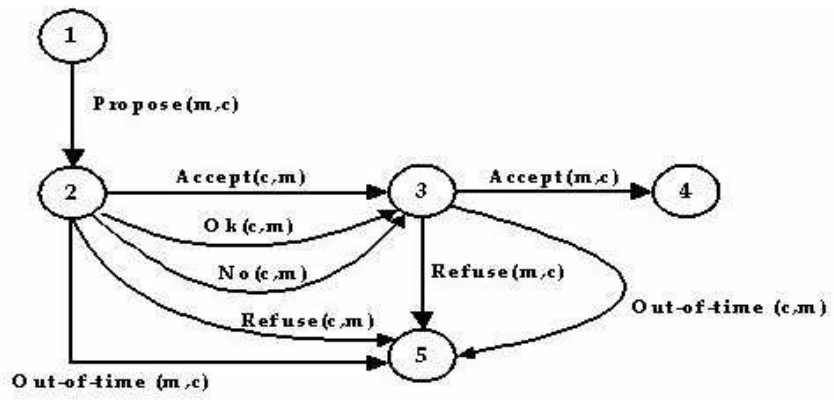

Figure 1: State transition graph of the negotiation between the manager $(\mathrm{m})$ and a candidate $(\mathrm{c})$

\subsection{The evaluation phase}

After receiving the candidates' responses, the manager evaluates and classifies them according to their importances. The evaluation is made using a multicriterion analysis. This phase includes two stages. The first one deals with the evaluation of the criteria and the second one with the aggregation of the evaluations.

\section{Criterion evaluation}

In this stage, the manager evaluates agents which sent their proposals according to various criteria. In [11], the author defines a whole of interesting criteria for the selection of the most adequate partner. We select those which seem most significant to our context. We have classified the criteria into three categories:

(a) Criteria related to the partner

- the cooperation degree of the candidate agent with the manager (D1) 
- the cooperation degree of the manager with the candidate agent (D2)

- the quality of the relation (D3) deduced from three others subcriteria:

(i) respect of the allowed time for achieving the subtask (S1)

(ii) the quality of achieving the subtask (S2).

(iii) Honesty with regard to the profit distribution after the last experiment (S3)

- the experiment in the cooperation:

(i) the cooperation number carried out by the partner (A1)

(ii) the cooperation number carried out by the partner without interruption (A2).

(b) Criteria related to the cooperation

- the capital (C1).

- the technical capacity (C2).

- the technological competencies (C3)

- the existence of a single capability (C4).

(c) Criteria related to the candidate agent proposal The candidate must specify in its acceptance message the time $T$ and the cost $C$ for achieving the subtask.

\section{The aggregation stage}

After the evaluation of the different criteria, the manager incorporates the evaluations associated with all the criteria in order to have an overall estimate of each agent. In CFoTACIS, the aggregation of the criteria is used in two cases:

(i) For the trust quantification: The trust $e$, which results from the current relation with the agent partner, is carried out using the equation

$$
e=p_{1} * S_{1}+p_{2} * S_{2}+p_{3} * S_{3}
$$

Where $p_{1}, p_{2}$, and $p_{3}$ are the weights that the agent must specify and $p_{1}+p_{2}+p_{3}=1$.

(ii) For the evaluation of each candidate: the aggregation operator is defined using a deviation at an aspiration point which gathers the preferred values of the criteria [12]. The evaluations are represented using a vector named $b$. The deviation $b$ to the aspiration point $a$ is defined by the relation.

$$
\operatorname{deviation}(a, b)=\operatorname{Max}_{j=1 . . p}\left(\lambda_{j}\left(a_{j}-b_{j}\right)\right)
$$

With $\lambda_{\mathrm{j}}=1 /\left(\right.$ Ideal $_{\mathrm{j}}$ - AntiIdeal $\left.\mathrm{j}_{\mathrm{j}}\right)$ and $p$ is the number of criteria. Ideal is a vector which gathers the maximal criteria values of the evaluation vector and Antildeal is a vector which gathers the minimal criteria values of the evaluation vector.

The best evaluation $\mathrm{d}^{*}$ is that which minimizes the deviation to the aspiration point:

$$
d^{*}=\min \{\operatorname{deviation}(a, b)\}
$$

For well explaining the evaluation mechanism, let us consider the following example. Agents Agl,

\begin{tabular}{|c|c|c|c|c|c|c|c|c|c|}
\hline Agents & \multicolumn{2}{|l|}{$\mathrm{Ag} 1$} & \multicolumn{2}{|l|}{ Ag2 } & \multicolumn{2}{|l|}{ Ag3 } & \multirow[t]{2}{*}{ Ideal } & \multirow[t]{2}{*}{ antiIdeal } & \multirow[t]{2}{*}{$\lambda$} \\
\hline Criterion & evall & $\lambda$ (Ideal-eval1) & eval2 & $\lambda$ (Ideal-eval2) & eval3 & $\lambda($ Ideal-eval3) & & & \\
\hline Criterion1 & 0.18 & 0 & 0.10 & 1 & 0.14 & 0.50 & 0.18 & 0.10 & 12.5 \\
\hline Criterion2 & 0.16 & 0 & 0.12 & 1 & 0.15 & 0.25 & 0.16 & 0.12 & 25 \\
\hline Criterion3 & 0.10 & 1 & 0.18 & 0 & 0.15 & 0.375 & 018 & 0.10 & 12.5 \\
\hline deviation & \multicolumn{2}{|l|}{1} & \multicolumn{2}{|l|}{1} & \multirow{2}{*}{\multicolumn{2}{|c|}{0.50}} & & & \\
\hline $\mathrm{d}^{*}$ & \multicolumn{4}{|l|}{0.50} & & & & & \\
\hline
\end{tabular}
$A g 2$, and $A g 3$ are evaluated according only to the criteria criterion1, criterion2, and criterion 3 in the vectors eval1, eval2, and eval3 (table 1). 
Table 1: An illustrative example of the adopted aggregation operator

\subsection{The finalization phase}

After evaluating agents' proposals, the manager classes agents in a list according to their deviations. The manager sends the agent which is at the head of the list a message for inviting it to join the coalition; else it contacts the next agent in the list, and so on.

The coalition members merge their deviation vectors to have a unified view of the candidates. This merger allows the coalition members to have supplemented information since agents have only a partial vision of their environment. The merger is carried out by the minimum operator. Let us consider an example showing how to make the unified evaluation vector. Agent $A g 3$ is evaluated by the members $A g 1$ (eval1) and $A g 2$ (eval2) w.r.t. to the eleven (11) criteria (see section 3.3.1).

$$
\begin{aligned}
& \text { eval1 }=(0.32,0.56,0.88,20,10,2000,1500,5,1,0.53,0.23) \\
& \text { eval2 }=(0.56,0.12,0.68,20,10,2000,1500,5,1,0.53,0.23)
\end{aligned}
$$

The unified deviation vector is

$$
\text { eval }=(0.32,0.12,0.68,20,10,2000,1500,5,1,0.53,0.23)
$$

Thus, for each subtask, the coalition members together try to choose the adequate partner.

The negotiation process will be finished when the coalition size is reached, and therefore, the coalition members start the execution of the tasks. Hence, with CFoTACIS, the decision of the coalition structure is collective.

\section{The CFoTACIS Properties}

The proposed model presents several advantages. The choice of the partners is carried out by the coalition members thanks to the unification process of agents' estimates carried out by each member. The adopted aggregation operator does not authorize the compensation, which permits to choose a partner which has acceptable values for all the criteria. CFoTACIS ensures the equity property because it offers the same chances to the candidates by limiting the time of the binary negotiations. When the candidate specifies its waiting time, this avoid the coalition members to send message to an agent that is no more waiting. Consequently, a considerable profit of time will be obtained. When a partner disengages, it is obvious that it will be sanctioned. This sanction is expressed by the A2 parameter (see section 3.3.1.a). In this case, the coalition members have two alternatives for replacing the disengaged agent. They propose the subtask to the coalition members to cheek whether it exists a partner which is interested to achieve it. Therefore, a new negotiation process is avoided. The second alternative is applied when there are not members which are interested. The coalition is obliged to start a new negotiation process to find another partner. Finally, if the manager could not find partners or the required size of the coalition is not reached; the negotiation process will be remade.

\section{Experimental results}

We have implemented CFoTACIS using JADE 3.0 (Java Agent DEvelopment). We have compared its performances with those of a similar model [3]. We remind that in [3], the author proposed two 
negotiation models. We are interesting to the one dedicated to egoistic agents considering the rationality of our agents.

We have made several series of experiments. In this paper, we show the effect of varying the agents' and subtasks' number on the global time of the negotiation process (figures 2 and 3 ).

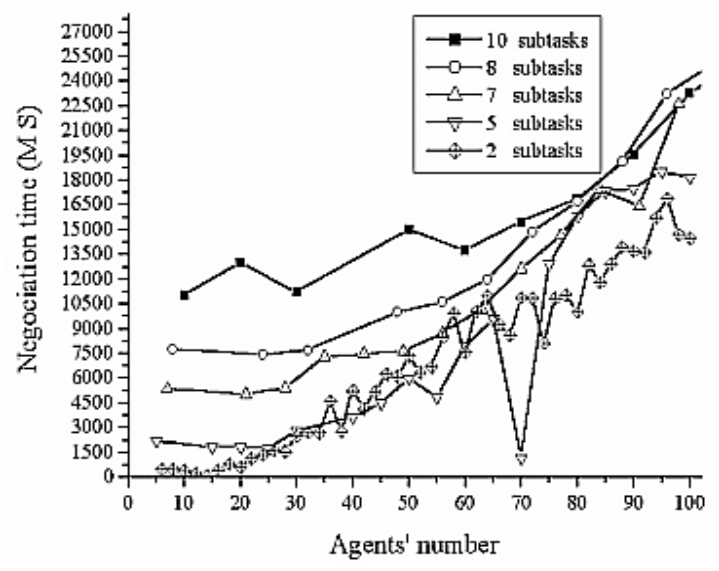

Figure 2: The negotiation time versus the agents' number for different subtasks' number in CFoTACIS

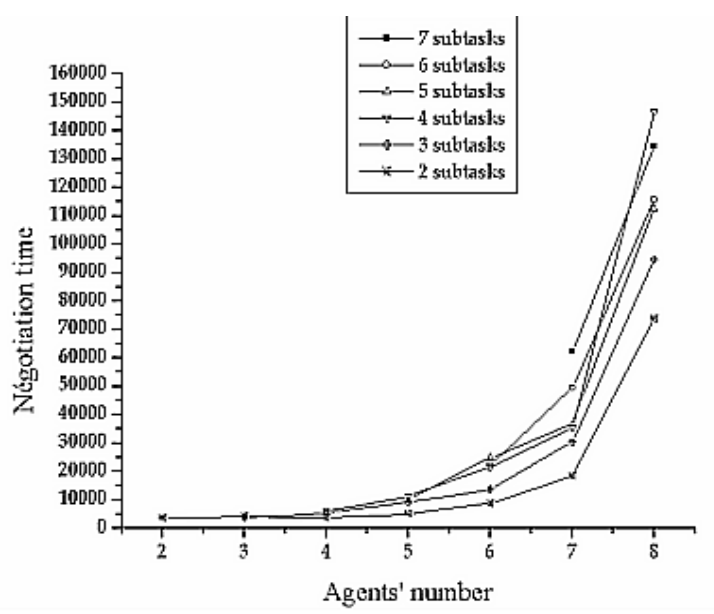

Figure 3: The negotiation time versus the agents' number for different subtasks' number in the model proposed in [3]

In each negotiation process, we fixe the subtasks' number and we increase the agents' number to finally deduce the consumed time. The graphs presented in this section are drowning using the Origin 6.0 software.

On the graph of figure 2 (CFoTACIS), we observe that we could vary agents' number from 2 to 100 and subtasks' number from 2 to 10 . Whereas, on the figure 4 (the comparative model), we could vary agents' number only from 2 to 8 and subtasks' number only from 2 to 7 . Therefore, we deduce that the CFoTACIS' scalability is stronger than the one of the comparative model. One reason of this result is that in the comparative model, all agents are in competition for the coalition formation. So, if we have $" n "$ agents in the system, we have also " $n$ " parallel negotiations which cause a scheduling problem in the system. On the other hand, we observe that the negotiation time in CFoTACIS increases as agents' and 
subtasks' number increase. But this increase stills reasonable and very small in comparing it with the one of the comparative model. This is due to the weak scalability of the last one. Also, in the comparative model, there is no limitation of the negotiation time between agents

\section{Summary and Conclusions}

In this paper, we have proposed a coalition formation-based negotiation model for the cooperative information agent-based systems, named CFoTACIS. The proposed model is inspired from the reality of the cooperation between enterprises. It includes four phases, the initialization, the negotiation, the evaluation, and the finalization. The essential properties of CFoTACIS are its consideration of the preferences of the coalition members for choosing the future partners. It does not apply any authority on agents and ensures the equity by limiting the time of the binary negotiations due to the absence of the cycles in the proposed negotiation protocol. However, it does not ensure the reach of a consensus but tries to encourage agents to cooperate. The simulation results show that in CFoTACIS the spent time for the negotiation is reasonable and the messages' number is deterministic. These results have been confirmed by comparing the CFoTACIS' performances with those of the negotiation model proposed in [3].

In the future work, we will improve CFoTACIS so that it will ensure the reach of a consensus. Also, we will adopt it in other application fields like e-commerce.

\section{References}

[1] L. Putnam, M. S. Pool, "Conflict and Negociation In Handbook of Organizational Communication: An Intersciplinary Perspective,” F.M.Jablinetal, Eds. Saga Newbury Park, pp. 549-599, California, 1987.

[2] T. Sandholm, "An Algorithm for Optimal Winner Determinism in Combinatorial Auctions," In Proc. of the 16th Int. Joint Conf. on AI, Stockholm, Sweden, 1999.

[3] S. Aknine, "Modèles et méthodes de Coordination des Systèmes Multi Agents," Thesis of Doctorate, University Paris IX Dauphine-UFR Science of the Organizations, December 2000.

[4] N. Zarour, M. Boufaida, and L. Seinturier, "A Negotiation Framework for Organizational Information Systems," Int. Journal of information Technology and Decision Making (IJITDM). World Scientific Publishing Co, , vol. 3, nř2, pp. 213-238, June 2004.

[5] A. E. F. Saghrouchni and S. Haddad, "Recursive Model for Distributed Plannin," In Proc. of the 2nd Int. Conf. On Multi-Agent Systems (ICMAS'96),, AAAI Press. Kyoto, Japan, 1996.

[6] C. Brooks and E. Durfee, "Congregating and Market Formation," Proc. of the 1st Int. joint conf. on Autonomous Agents and Multiagent systems, , ACM Press, pp 96-103, 2002.

[7] A. E .F. Saghrouchni and G. Vauvert, "Formation de Coalitions pour Agents Rationnels," In Proc. of ILIPN'2000. VIIIth days of LIPN. Multi-Agent Systemes and Formal Specification and Software Technologies ,,Viltaneuse, France 09, 11-12, 2000.

[8] L. K. Soh, C. Tsatsouli, and H. Servey, "A Satisficing Negotiated and Learning Coalition Formation Architecture," In V.Lesser, C.Ortiz, and M.Tambe, editors, Distributed Sensor Networks: a Multi-Agent perspective,,Kluwer Academic Publishers, 04, 25-27, 2001.

[9] L. Larson and T. Sandholm, "Anytime Coalition Structure Generation: an Average Case Study," Journal of Experiments and Therotical Al,11, 1-20, 2000.

[10] Vassileva, J. Breban and S. Horsch, "Agent Reasoning Mechanism for Long-Term Coalitions Based on Decision Making and Trust," Computational Intelligence, 18, 4, Special Issue on Agent Mediated Electronic Commerce, , pp 583-595, Nov. 2002. 
[11] C. Li and K. Sycara, "A Stable and Efficient Scheme for Task Allocation via Agent Coalition Formation," www.2.cs.cmu.edu/ softagents/papers/CCO3.pdf, 2003.

[12] ] N. R. Jennings, P. Faratin, P. Johnson, M. J. O’Brien, and M. Wiegand, "Using Intelligent Agents to Manage Business Processes," Practical application of intelligent Agents and Multi-Agents technology, PAAM 96,1999.

Nacer eddine Zarour, Sabrina Bouzidi

University Mentouri of Constantine

LIRE Laboratory, 25000 Algeria

E-mail: nasro_zarour@yahoo.fr, sab_bouzidi@yahoo.fr 